\title{
Wireless Image Transmission Interference Signal Recognition System Based on Deep Learning
}

\author{
Zhijun Guo $\mathbb{D}^{1,2}$ and Shuai Liu ${ }^{1,2}$ \\ ${ }^{1}$ Institute of Information Science and Technology, Hunan Normal University, Changsha 410081, China \\ ${ }^{2}$ Hunan Xiangjiang Artificial Intelligence Academy, Changsha 410081, China \\ Correspondence should be addressed to Zhijun Guo; guozhijun@hunnu.edu.cn
}

Received 18 September 2021; Accepted 13 November 2021; Published 16 December 2021

Academic Editor: Deepak Gupta

Copyright (C) 2021 Zhijun Guo and Shuai Liu. This is an open access article distributed under the Creative Commons Attribution License, which permits unrestricted use, distribution, and reproduction in any medium, provided the original work is properly cited.

\begin{abstract}
In the process of wireless image transmission, there are a large number of interference signals, but the traditional interference signal recognition system is limited by various modulation modes, it is difficult to accurately identify the target signal, and the reliability of the system needs to be further improved. In order to solve this problem, a wireless image transmission interference signal recognition system based on deep learning is designed in this paper. In the hardware part, STM32F107VT and SI4463 are used to form a wireless controller to control the execution of each instruction. In the software part, aiming at the time-domain characteristics of the interference signal, the feature vector of the interference signal is extracted. With the support of GAP-CNN model, the interference signal is recognized through the training and learning of feature vector. The experimental results show that the packet loss rate of the designed system is less than $0.5 \%$, the recognition performance is good, and the reliability of the system is improved.
\end{abstract}

\section{Introduction}

The emergence of wireless image transmission technology makes it convenient, fast, accurate, and real time for people in need to obtain remote image information, which provides convenience for special industry applications [1]. In recent years, the related technologies of wireless image transmission system have developed rapidly, but there are still some limitations, such as limited transmission bandwidth, inflexible transmission mode, and communication and image transmission quality assurance mechanism. Therefore, the research on these related aspects has extremely important social and economic value [2-4].

The existing wireless image acquisition and transmission system can only be used in the place where the line is laid in advance, and its application range is greatly limited. At present, many of them use GPRS/CDMA (General Packet Radio Service and Code Division Multiple Access) technology to achieve wireless image acquisition and transmission system, which uses the cellular network transmission mode, so the transmission signal must rely on the base station. In a bad environment, such as catastrophic weather and special circumstances, when the base station fails, the whole communication network will be destroyed. Even if it is not damaged, there will be a lot of interference in the transmission signal, which will affect the performance of wireless image transmission [5-7]. Although the JPEG image can be received in any area covered by the signal, the transmission of image data is not very stable. In addition, the user has to bear the communication cost. The existing underlying communication protocol of the Internet of things consumes too much power in the application of wireless image sensing system and cannot support sudden big data transmission (image data transmission) [8-10]. Therefore, according to the network transmission requirements of the above different application fields, the research and development of a low-power, low-cost, self-relaying narrow-band communication network system for rapid deployment without power 
supply infrastructure will help to rapidly promote the scale of related Internet of things applications and promote the application in intelligent power grid, intelligent transportation, industry and automation control, etc. Application promotion of IoT in the field of environment and safety inspection [11-13]. The realization of this reliable network communication is inseparable from the identification of interference signals. Only by accurately identifying the interference signals in wireless transmission can we further ensure the quality of wireless transmission.

Signal recognition technology originated in the early last century; with the early application in radio signal interference, information interception, and other military-related fields, after long-term research and development, the relevant signal recognition technology has been proposed. The early recognition technology is relatively primitive and relies more on manual work. Nowadays, the research on signal recognition is mostly modulation pattern recognition, and this traditional recognition pattern is far from enough [14]. In recent years, signal recognition technology has a very good development, and professionals have improved. After the system design is completed, the traditional identification system is introduced, and a large number of comparative experiments are carried out image transmission, the traditional identification system includes Fang et al., which integrates the multidimensional domain characteristic parameters of typical interference signals such as singletone interference, broadband noise interference, multitone interference, and sweep interference, such as time domain, frequency domain, and transform domain [15]. The jamming signal recognition method based on decision tree algorithm and the range velocity composite deception jamming recognition technology based on space-time frequency feature fusion are proposed by Yang et al. This method completes the jamming signal recognition by extracting the spatial and frequency domain features of echo traces, respectively, and fusing the extracted spatial and frequency domain features in time domain [16]. These traditional recognition systems can meet some basic recognition requirements. However, as the image attributes become more and more complex, the service signal adopts more complex multiple modulation, so it is difficult for the recognition system to accurately identify the target signal, and the reliability of the system needs to be further improved. In order to solve the above problems and improve the effect of target signal recognition, a wireless image transmission interference signal system based on deep learning is proposed in this paper. In the hardware part, stm $32 \mathrm{f} 107 \mathrm{vt}$ and si4463 are used to form a wireless controller to control the execution of each instruction. In the software part, aiming at the timedomain characteristics of the interference signal, the feature vector of the interference signal is extracted. With the support of GAP-CNN model, the interference signal is recognized through the training and learning of feature vector. Through the above steps, the identification of interference signals is completed, and through the experimental link, the effect of the design system is proved, in order to provide some help to improve the identification effect of interference signals.

\section{Hardware Design of Wireless Image Transmission Interference Signal Recognition System Based on Deep Learning}

According to the characteristics of wireless image transmission, the wireless network controller is designed in the hardware design part of the system. Wireless network controller (WNC) consists of "micro control unit (MCU)" and "wireless module." The main function of micro control unit is to control business logic and implement wireless communication protocol and network communication protocol. Among them, the role of wireless module is to complete the wireless signaling and data transceiver function $[17,18]$. The wireless module and wireless protocol interact with WIS to complete the transmission of image data from WIS to WNC. WNC interacts with the "image monitoring platform" through Ethernet to execute the commands issued by it. WNC plays a role of bridge between the "image monitoring platform" and WIS and completes the conversion of information between wireless and network transmission media [19].

Wireless network controller (WNC) is composed of STM32 series $107 \mathrm{MCU}$, wireless module, and Ethernet PHY chip. The MCU of STM32F 107 has an Ethernet controller, which only needs to expand PHY chip of Ethernet to realize Ethernet communication. The hardware block diagram of the wireless network controller (WNC) is shown in Figure 1.

The WNC board controller is STM32F107VT, with the main frequency of $72 \mathrm{MHz}$ and the Ethernet MAC controller with $10 / 100 \mathrm{M}$ on the chip and is compatible with the MII and RMII interfaces. Through RMII interface, it can realize seamless connection with PHY chip DM9161CI. The network transformer HX2019 mainly enhances the anti-interference and load capacity of network port [20]. The SPI interface of WNC is connected with the wireless module. The system realizes airport communication, receives image data and uplink control signaling, and sends downlink control signaling.

MCU module is composed of the smallest system of STM32F107. Its function is connected with other modules through various interfaces to complete the control of other modules. The wireless module is a wireless transceiver module composed of SI4463 chip. It is connected with the MCU module through SPI interface. MCU controls the wireless module to send and receive packets through SPI to complete the task of wireless communication. Network interface module is composed of network controller in MCU and HX2019 chip. It is responsible for the basic sending and receiving of the network to complete the network communication task. The SD card is composed of card reader interface. Its function is to cache the image information collected from WIS to transmit the image upward when the network is not busy.

According to the above contents, the power consumption of main devices is counted, and the WNC power supply is designed. The details are shown in Table 1.

At present, the commonly used power management chips are linear power supply (LDO power supply) and DCDC switching power supply. So far, the hardware design of the identification system has been completed, and on this basis, the software part is designed. 


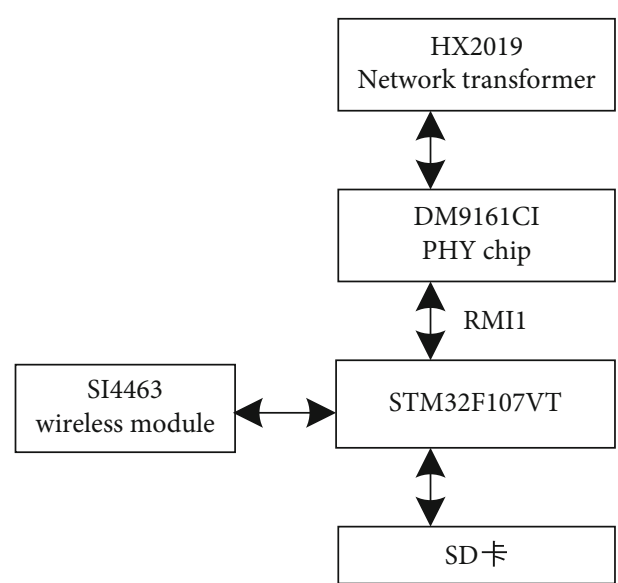

FIGURE 1: Hardware block diagram of wireless network controller.

Table 1: Power consumption of main components of WNC.

\begin{tabular}{lccc}
\hline Main chip & $\begin{array}{c}\text { Supply } \\
\text { voltage (V) }\end{array}$ & $\begin{array}{c}\text { Power } \\
\text { consumption } \\
(\mathrm{mW})\end{array}$ & $\begin{array}{c}\text { Supply } \\
\text { current (mA) }\end{array}$ \\
\hline STM32F107VT & 3.3 & 350 & 106.06 \\
DM9161CI & 3.3 & 290 & 87.88 \\
S14463 & 3.3 & 270 & 81.82 \\
Other & 3.3 & 100 & 30.30 \\
\hline
\end{tabular}

\section{Software Design of Wireless Image Transmission Interference Signal Recognition System Based on Deep Learning}

3.1. Feature Extraction of Interference Signal. In deep learning, supervised $\mathrm{CNN}$ architectures for classification, such as AlexNet, GoogLeNet, and ResNet, all take the Sofhnax layer as the output layer, which is connected with the convolution layer or full connection layer. The input of Softmax layer is the feature vector of the original input signal $y(n)$, so it can be considered that the function of the convolution layer in $\mathrm{CNN}$ is to map $y(n)$ to the feature space, that is, to extract the feature vector. In the convolution layer, each convolution kernel can be considered as a linear system for extracting a certain feature. But before the network training, the operation parameters of the whole system are unknown. Therefore, the weight parameters of convolution kernel are randomly initialized, and the parameters of the system can only be optimized and updated by BP algorithm to reduce the value of the objective function. After the training, the system can be used to extract the input features. The concatenation of convolution layers makes the input signal $y(n)$ be abstractly mapped layer by layer, and finally, the feature vector needed by the classifier is obtained. But before network training, the operating parameters of the whole system are unknown. Therefore, the weight parameters of convolution kernel are also initialized randomly. In this case, in order to reduce the value of the objective function, the system parameters need to be optimized and updated by the BP algorithm. After training, the system can be used to extract the input features, and then, the input signal $y(n)$ is abstractly mapped layer by layer through the superposition of convolution layers, so as to finally obtain the feature vector required by the classifier, so as to overcome the influence of unknown operating parameters.

In the process of feature extraction of jamming signal, the time-domain feature of jamming signal is taken as the extraction target. It is assumed that $x(n)$ is the discrete time-domain sequence of jamming signal, and the characteristic parameter reflecting the steepness of amplitude distribution of time-domain signal $x(n)$ is the time-domain moment kurtosis. The expression is as follows:

$$
\alpha_{1}=\frac{p(x(n)-\varepsilon)}{\eta^{4}}
$$

In the formula, $\varepsilon$ is the mean value of time-domain signal and $\eta$ is the standard deviation of time-domain signal. It can be seen from formula (1) that the characteristic parameter $\alpha_{1}$ is a fourth-order statistic and generally takes the kurtosis of moment in the time domain of normal distribution signal as the comparison standard. If the kurtosis of the moment in time domain is less than 3, the timedomain distribution of the signal is relatively flat, which is called flat peak distribution. If the kurtosis of the timedomain moment is greater than 3 , the time-domain distribution of the signal is relatively steep, which is called the peak distribution. In order to further characterize the fluctuation of amplitude distribution in time domain, the normalized amplitude standard deviation of target signal is calculated. The smaller the value, the distribution is relatively balanced, otherwise, the greater the amplitude of the signal changes. The calculation formula is as follows:

$$
\begin{gathered}
x_{0}(u)=\frac{x(u)-\varepsilon}{\varepsilon} \\
\alpha_{2}=\left(\frac{1}{N-1} \sum_{i=1}^{n}\left(\left|x_{0}(u)\right|-\frac{1}{N} \sum_{i=1}^{n}\left|x_{0}(u)\right|\right)^{2}\right)^{1 / 2} .
\end{gathered}
$$

In the formula, $x_{0}(u)$ represents the normalized value of amplitude sequence $x(n)$, and $N$ represents the number of sequences. After the target is determined, the feature vector of interference signal is extracted by encoder and decoder. In the encoder stage, after the convolution layer mapping, the signal is downsampled through the pooling layer to compress the size of the output feature map. After several times of the same operation, the convolution layer at the bottom is reached, and the output at the bottom can be regarded as the abstraction of the original input signal $y(n)$. In the decoder stage, these abstract features are sampled continuously by the deconvolution layer and finally reach the top output layer. The goal of CAE is to output and input consistently, and its extraction process can be described as "compress before refactoring." After obtaining the feature vector of the interference signal, the interference signal is recognized in the wireless transmission signal. 
3.2. Identify Jamming Signal. In order to recognize the interference signal by deep learning, it is necessary to generate the signal training set before recognition, so as to provide the necessary data for signal recognition. Moreover, the signal training set is mainly completed through software simulation and hardware acquisition. The interference signals to be identified are commonly used digital modulation signals, including MPSK, MQAM, MFSK, and GMSK modulation types. The hardware acquisition adopts NI-USRP 2920 software radio signal receiving and receiving platform, which is in the same way as the real communication to modulate the code elements, and then, it is filtered and mixed to high frequency and transmitted. At the receiving end, the captured signal is mixed to it by analog frequency and then is collected and processed by digital downconversion. Finally, the complex baseband signal is obtained. The signal collected by hardware contains all the modulation information of the signal and is very close to the characteristics of the signal in real communication. However, due to the limitations of NI-USRP equipment, there are many problems in signal acquisition, such as non-real-time and complicated operation, and it is difficult to simulate various complex outdoor channels. Therefore, in order to make up for these shortcomings, this paper also uses software simulation to supplement the training set data. The training set includes the baseband signal after shaping filtering at the transmitter, the baseband signal after digital downconversion at the receiver, and the modulation type. After the Recovery-Net training, the collected output data is stored in the training set.

In the recognition of interference signal, $\mathrm{CNN}$ and GAPCNN models in deep learning are combined. In order to make the structure of the model concise and learn the characteristics of the interference signal well, we built the CNN and GAP-CNN model structure. The model has the following characteristics: in the convolution process, the space size becomes smaller and the output depth increases layer by layer. In deep learning, more convolution kernels can learn more features, but the number of convolution kernels should be kept within a reasonable range, because the unreasonable number of convolution kernels will interfere with the recognition and affect the recognition effect. When the size of convolution kernel is fixed, the number of convolution kernel must be proportional to the depth of input and output of the convolution layer. Under the above requirements, the convolution pooling layer in $\mathrm{CNN}$ is usually connected with one or more fully connected layers, and the fully connected layer transforms the input feature spectrum into vectors to complete the dimension reduction of the input and provide input for subsequent recognition. However, when the parameters are too large, it is difficult for the CNN network to support the identification service alone. Therefore, in order to reduce the network parameters and the risk of over fitting, the convolution layer is used to replace the full connection layer, and the large convolution core is used to connect with a small number of nodes in the back layer. Gap is not directly used to replace the full connection layer. In the design, GAP-CNN layer is introduced to replace the flattening layer to reduce the input quantity of the full con-
TABle 2: Parameter configuration of NI-USRP 2920.

\begin{tabular}{lcc}
\hline $\begin{array}{l}\text { Parameter } \\
\text { number }\end{array}$ & Project & Parameter \\
\hline Q01 & I/Q sampling rate & $500 \mathrm{k} \mathrm{sample} / \mathrm{s}$ \\
Q02 & Oversampling multiple & 4 \\
Q03 & Symbol rate & $125 \mathrm{k} \mathrm{symbol} / \mathrm{s}$ \\
Q04 & Carrier frequency & $915 \mathrm{MHz}$ \\
Q05 & Transmit/receive gain & $10 \mathrm{~dB} / 20 \mathrm{~dB}$ \\
Q06 & Transmit/receive & $T \times 1 / R \times 2$ \\
Q07 & antenna & 0.4 \\
Q08 & Roll-off factor & 20 \\
Q09 & Filter order & Root raised cosine \\
\end{tabular}

nection layer. Other structures are designed by CNN. After the design of the GAP-CNN model structure, the training set is used as the input to complete the learning process. Then, the target to be identified is input into the model to complete the recognition of interference signal of wireless image transmission. So far, the design of wireless image transmission interference signal recognition system based on deep learning is completed.

\section{Experimental Study on the Interference Signal Recognition System of Wireless Image Transmission Based on Deep Learning}

4.1. Building Experimental Platform. Through the construction of wireless communication transceiver platform, the verification of wireless image transmission interference signal recognition system is carried out in the real environment. NI-USRP 2920 is based on the public version of software radio platform USRP Radio by NI instrument company. It is a part of the external circuit transformation. NIUSRP hardware has a common software-defined radio (SDR) architecture. In its FPGA digital signal processing logic, the communication transmitter modulates the user data into digital baseband data, and the output becomes ana$\log$ baseband signal I/Q through high-speed DAC. After the high-order harmonics and spurious are filtered by low-pass filter, the analog baseband signal is up converted to the carrier frequency $915 \mathrm{MHz}$ by analog RF quadrature, and the communication transmission is completed by power amplification and antenna. At the communication receiving end, the weak wireless communication signal received by the antenna is modulated by low-noise amplifier, and then, the analog RF orthogonal downconversion is completed. Output $I / Q$ baseband signal to high-speed ADC; quantize I/Q data stream to FPGA digital signal processing logic. The FPGA digital signal processing logic is used to realize the digital downconversion (DDC) at the receiving end and the digital upconversion (DUC) at the transmitting end. Through the experimental platform, the interference signal and normal 
TABLE 3: Experimental signal parameters.

\begin{tabular}{lcccccc}
\hline & Center frequency & Sampling rate & Code rate & Signal intensity & Modulation depth & Inter frequency \\
\hline AM signal & 300 & 192 & - & -40 & $50 \sim 100$ & - \\
FM signal & 300 & 192 & - & -40 & - & $4-10$ \\
PSK signal & 300 & 192 & $20-80$ & -40 & - \\
\hline
\end{tabular}

TABLE 4: Experimental results of packet loss rate of different identification systems.

\begin{tabular}{|c|c|c|c|c|}
\hline & Transmission interval & Packet loss rate & Number of images & Number of images in question \\
\hline \multirow{3}{*}{ Traditional identification system 1} & $3 \mathrm{~s}$ & $12.5 \%$ & 1000 & 12 \\
\hline & $5 \mathrm{~s}$ & $14.6 \%$ & 1000 & 9 \\
\hline & $8 \mathrm{~s}$ & $17.2 \%$ & 1000 & 14 \\
\hline \multirow{3}{*}{ Traditional identification system 2} & $3 \mathrm{~s}$ & $9.2 \%$ & 1000 & 4 \\
\hline & $5 \mathrm{~s}$ & $8.7 \%$ & 1000 & 8 \\
\hline & $8 \mathrm{~s}$ & $11.6 \%$ & 1000 & 9 \\
\hline \multirow{3}{*}{ The proposed recognition system } & $3 \mathrm{~s}$ & $0.3 \%$ & 1000 & 0 \\
\hline & $5 \mathrm{~s}$ & $0.4 \%$ & 1000 & 0 \\
\hline & $8 \mathrm{~s}$ & $0.2 \%$ & 1000 & 0 \\
\hline
\end{tabular}

signal of wireless image transmission can be effectively distinguished.

In the experiment, the transceiver parameters of NIUSRP 2029 digital transceiver that need to be adjusted are shown in Table 2.

The table, respectively, lists the specific parameters required by the equipment as signal generator and signal receiver, including the carrier frequency, symbol rate, modulation type, transceiver gain, and other parameters of the signal. In the experiment, the receiver is set to display and store the $I / Q$ signal sequence to the hard disk every $50 \mathrm{~ms}$; then, the sequence is processed into the format required by the subsequent work by the software and finally added to the data set.

4.2. Generation of Data Set. The experimental data used in the experiment are all generated and collected by the signal receiving and receiving equipment in the previous section. The experiment collects six kinds of experimental data: AM modulation signal, FM modulation signal, BPSK modulation signal, QPSK modulation signal, 8PSK modulation signal, and noise. Among them, the parameter range set in the signal generator to generate 5 modulation signals is shown in Table 3.

For the binary signals received by the receiver, the timedomain data set is obtained by reading every 16-bit binary signal as a real number. The SNR is added or interfered by the data specified by MATLAB simulation program, which makes the original regular signal start to spread randomly after noise.

4.3. Experimental Results and Analysis of Packet Loss Rate. After the preparation of experimental data, two widely used traditional recognition systems 1 and 2 (literature [15] and literature [16] systems) are compared with the designed system. Under the same experimental conditions, the packet loss rate experiment and the recognition performance experiment are carried out, respectively. In the two groups of experiments, the data set in the above is taken as the target.

In the experiment of packet loss rate, different wireless image transmission intervals are set. In the process of identifying interference signals, the third-party plug-in is used to supervise the working process of the system. After the transmission, the packet loss rate, the total number of images, and the number of images with problems are output. The more the number of images with problems is, the higher the packet loss rate is. The experimental results are shown in Table 4.

According to the data in Table 3, with the change of transmission interval, the packet loss rate of the system has different changes. The highest packet loss rate of the two traditional recognition systems reaches $17.2 \%$, and a certain number of problematic images appear, which indicates that the transmission reliability of the system is not guaranteed in the recognition process. In contrast, the packet loss rate of the designed recognition system based on deep learning is less than $0.5 \%$, and there is no problem image when processing the same number of images, which indicates that the transmission reliability of the system is guaranteed. The reason why the design system has such a good effect is that the design system optimizes and updates the system parameters through the BP algorithm. After training, the system can be used to extract the input features. Then, through the superposition of convolution layers, the input signals are abstractly mapped layer by layer, and finally, the feature vector required by the classifier is obtained, which not only overcomes the influence of unknown operating parameters. It also ensures the integrity of information transmission and reduces the packet loss rate.

4.4. Experimental Results and Analysis of Recognition Expressiveness. Different recognition systems are used to 


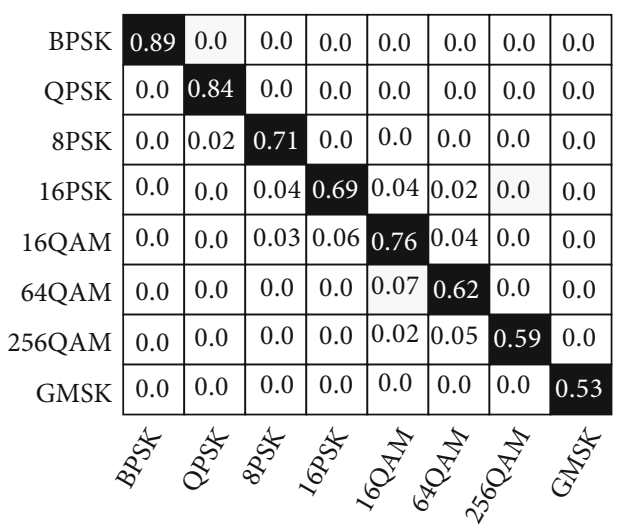

(a) Experimental results of traditional recognition system 1

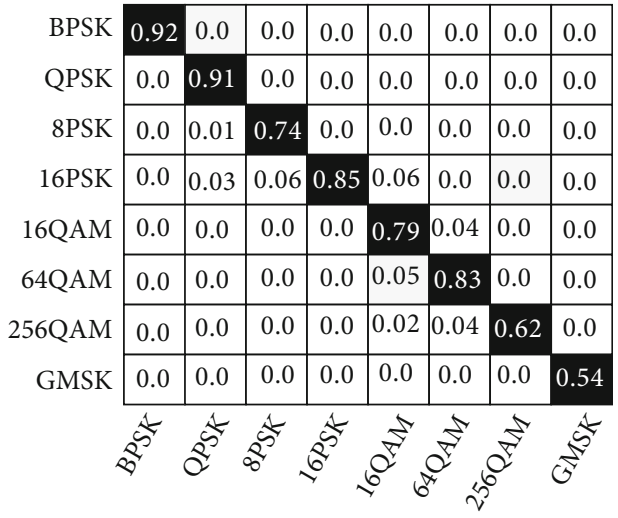

(b) Experimental results of traditional recognition system 2

\begin{tabular}{|c|c|c|c|c|c|c|c|c|}
\hline BPSK & 1.0 & 0.0 & 0.0 & 0.0 & 0.0 & 0.0 & 0.0 & 0.0 \\
\hline QPSK & 0.0 & 0.98 & 0.0 & 0.0 & 0.0 & 0.0 & 0.0 & 0.0 \\
\hline 8PSK & 0.0 & 0.0 & 0.95 & 0.0 & 0.0 & 0.0 & 0.0 & 0.0 \\
\hline $16 \mathrm{PSK}$ & 0.0 & 0.0 & 0.0 & 0.92 & 0.0 & 0.0 & 0.0 & 0.0 \\
\hline 16QAM & 0.0 & 0.0 & 0.0 & 0.0 & 0.94 & 0.0 & 0.0 & 0.0 \\
\hline 64QAM & 0.0 & 0.0 & 0.0 & 0.0 & 0.0 & 0.96 & 0.0 & 0.0 \\
\hline 256QAM & 0.0 & 0.0 & 0.0 & 0.0 & 0.0 & 0.0 & 0.97 & 0.0 \\
\hline GMSK & 0.0 & 0.0 & 0.0 & 0.0 & 0.0 & 0.0 & 0.0 & 0.91 \\
\hline
\end{tabular}

(c) Experimental results of the proposed recognition system

FIGURE 2: Experimental results of recognition expressiveness of different recognition systems.

process the signals in the experimental data set, and the recognition performance of the recognition system is analyzed according to the recognition level of different types of signals. The signals in the experimental data set are completed by NI-USRP2920. Before the experiment, MATLAB is used to interfere with the signals, and then, different recognition systems are used to identify them. The recognition results are shown in the form of confusion matrix, and the specific content is shown in Figure 2.

Compared with the experimental results, it can be seen that BPSK and GMSK are the most easily recognized signal types, and their accuracy is the highest among all the results. The experimental result of the proposed recognition system is the best, reaching $100 \%$. Among other signals, the traditional recognition system has the worst recognition effect on 256QAM and GMSK signals, only about 50\%, while the recognition accuracy of the proposed recognition system is more than $90 \%$. And it can be seen from the results in the figure that the traditional recognition system has a certain deviation for the recognition of some signals, so it is difficult to correctly identify the signal type, but the proposed signal recognition system can maintain a more accurate recognition effect. This is because the design system not only uses STM32F107VT and SI4463 to form a wireless controller to control the execution of each instruction but also takes the time-domain characteristics of the interference signal as the target to extract the characteristic vector of the interfer- ence signal. With the support of the GAP-CNN model, the interference signal is recognized through the training and learning of the feature vector. Through multiple guarantees, the signal recognition effect is improved.

Combined with the experimental analysis of the system packet loss rate, it can be seen that the designed wireless image transmission interference signal recognition system based on deep learning is more reliable, and the performance of interference signal recognition is also very good. The overall reliability of the system is better than the traditional recognition system.

\section{Conclusion}

With the emergence of a large number of wireless communication signals, signal service identification has become a new requirement in the field of signal identification. In the field of wireless image transmission, signal recognition is very important. It is an important link to ensure the quality of image transmission. On this basis, based on a large number of literature research, a wireless image transmission interference signal recognition system based on deep learning is proposed, the development and design of the whole system are completed from two aspects of hardware and software, and a large number of comparative experiments are carried out. The reliability of the designed system is verified by experiments. This shows the effect and function of the 
system designed in this paper in the field of interference signal recognition. However, the interference signals are not classified during the design, which may affect the identification effect. In the next research process, we will conduct indepth research on this point to better improve the interference signal identification effect of the design system.

\section{Data Availability}

The data sets used and/or analyzed during the current study are available from the corresponding author on reasonable request.

\section{Conflicts of Interest}

It is declared by the authors that this article is free of conflict of interest.

\section{Acknowledgments}

This study was financially supported by the Natural Science Foundation of Hunan Province with No. 2020JJ4434 and Hunan Provincial Science and Technology Department Project Foundation (2018TP1018, 2018RS3065).

\section{References}

[1] X. LI, J. LI, Y. QU, and D. HE, "Semi-supervised gear fault diagnosis using raw vibration signal based on deep learning," Chinese Journal of Aeronautics, vol. 33, no. 2, pp. 418-426, 2020.

[2] S. Y. Lee, C. Tsou, and P. W. Huang, "Ultra-high-frequency radio-frequency-identification baseband processor design for bio-signal acquisition and wireless transmission in healthcare system," IEEE Transactions on Consumer Electronics, vol. 66, no. 1, pp. 77-86, 2020.

[3] C. F. Yee, M. Mohamad Isa, A. Abdullah al-Hadi, and M. K. Md Arshad, "Techniques of impedance matching for minimal PCB channel loss at 40 GBPS signal transmission," Circuit World, vol. 45, no. 3, pp. 132-140, 2019.

[4] A. L. Yi, L. S. Yan, H. J. Liu et al., "Modulation format identification and OSNR monitoring using density distributions in Stokes axes for digital coherent receivers," Optics Express, vol. 27, no. 4, pp. 4471-4479, 2019.

[5] W. P. Tang, A. Q. Wang, S. Ramkumar, and R. K. R. Nair, "Signal identification system for developing rehabilitative device using deep learning algorithms," Artificial Intelligence in Medicine, vol. 102, 2020.

[6] A. H. Tan and K. R. Godfrey, "Direct synthesis signal sets for multi-input system identification," Systems \& Control Letters, vol. 124, pp. 92-98, 2019.

[7] S. S. Miriyala and K. Mitra, "Deep learning based system identification of industrial integrated grinding circuits," Powder Technology, vol. 360, pp. 921-936, 2020.

[8] G. Singh, S. Agrawal, and B. S. Sohi, "Handwritten Gurmukhi digit recognition system for small datasets," Traitement $d u$ Signal, vol. 37, no. 4, pp. 661-669, 2020.

[9] X. D. Yan and X. G. Song, "An image recognition algorithm for defect detection of underground pipelines based on convolutional neural network," Traitement du Signal, vol. 37, no. 1, pp. 45-50, 2020.
[10] H. Qu, T. Feng, Y. Wang, and Y. Zhang, "AdaBoost-SCN algorithm for optical fiber vibration signal recognition," Applied Optics, vol. 58, no. 21, 2019.

[11] E. Agliari, F. Alemanno, A. Barra, M. Centonze, and A. Fachechi, "Neural networks with a redundant representation: detecting the undetectable," Physical Review Letters, vol. 124 , no. 2 , article $028301,2020$.

[12] R. Supekar and T. Peacock, "Interference and transmission of spatiotemporally locally forced internal waves in nonuniform stratifications," Journal of Fluid Mechanics, vol. 866, pp. 350-368, 2019.

[13] K. Takahashi, T. Yamada, and Y. Takemura, "Circuit parameters of a receiver coil using a Wiegand sensor for wireless power transmission," Sensors, vol. 19, no. 12, p. 2710, 2019.

[14] W. H. W. Hassan, S. M. Idrus, H. King, S. Ahmed, and M. Faulkner, "Idle sense with transmission priority in fibrewireless networks," IET Communications, vol. 14, no. 9, pp. 1428-1437, 2020.

[15] F. Fang, Y. Li, Y. Niu, and Y. Wang, "Interference signal recognition based on decision tree algorithm," Communication Technology, vol. 52, no. 11, pp. 2617-2623, 2019.

[16] L. Yang, X. Zhang, L. Li, and G. Wang, "Research on range velocity composite deception jamming identification technology based on space-time frequency feature fusion," Systems engineering and electronic technology, vol. 41, no. 12, pp. 2684-2691, 2019.

[17] A. Lund, S. J. Dyke, W. Song, and I. Bilionis, “Global sensitivity analysis for the design of nonlinear identification experiments," Nonlinear Dynamics, vol. 98, no. 1, pp. 375-394, 2019.

[18] T. F. Bernard Marie, D. Han, and B. An, "Pattern recognition algorithm and software design of an optical fiber vibration signal based on $\Phi$-optical time-domain reflectometry," Applied Optics, vol. 58, no. 31, pp. 8423-8432, 2019.

[19] R. Hercik, Z. Machacek, J. Koziorek, J. Vanus, M. Schneider, and W. Walendziuk, "Continuity detection method in binary image signal," Elektronika ir Elektrotechnika, vol. 26, no. 6, pp. 4-9, 2020.

[20] W. Lejmi, A. B. Khalifa, and M. A. Mahjoub, "A novel spatiotemporal violence classification framework based on material derivative and LSTM neural network," Traitement du Signal, vol. 37, no. 5, pp. 687-701, 2020. 